\title{
Nonideal Measurement Locations in Planar Near-Field Antenna Metrology
}

\author{
R. C. Wittmann, B. K. Alpert, and M. H. Francis \\ National Institute of Standards and Technology \\ 325 Broadway, Boulder, CO 80303-3328
}

\section{Introduction}

We introduce a near-field to far-field transformation method that relaxes the usual restriction that data points be located on a plane-rectangular grid [1]. It is not always practical or desirable to make uniformly spaced measurements; for example, the maintenance of positioning tolerances becomes more difficult as frequency is increased. Our method can (1) extend the frequency ranges of existing scanners, (2) make practical the use of portable scanners for on-site measurements, and (3) support schemes, such as plane-polar scanning, where data are collected on a nonrectangular grid.

Although "ideal" locations are not required, we assume that probe positions are known. (In practice, laser interferometry is often used for this purpose.) Our approach is based on a linear model of the form $\mathbf{A} \boldsymbol{\xi}=\mathbf{b}$ (see section 2). The conjugate gradient method is used to find the "unknown" $\boldsymbol{\xi}$ in terms of the "data" $\mathrm{b}$ (section 3). The operator $\mathbf{A}$ must be applied once per conjugate gradient iteration, and this is done efficiently using the recently developed unequally spaced fast Fourier transform [2], [3] and local interpolation (section 4). As implemented, each iteration requires $\mathcal{O}(N \log N)$ operations, where $N$ is the number of measurements. The required number of iterations depends on desired computational accuracy and on conditioning. In section 5 , we present a simulation that is based on actual near-field antenna data.

\section{The Model}

Consider a transmitting test antenna (located in the half space $z<0$ ) and a receiving probe (translated without rotation). According to Kerns's theory [4], the probe response $w(\mathbf{r})$ may be modeled as

$$
w(\mathbf{r})=\sum_{\nu \mu} \xi_{\nu \mu} \exp \left(i \mathbf{k}_{\nu \mu} \cdot \mathbf{r}\right), \quad z>0
$$

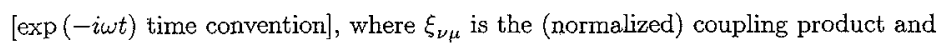

$$
\mathbf{k}_{\nu \mu}=\frac{\pi \nu}{L_{x}} \hat{\mathbf{x}}+\frac{\pi \mu}{L_{y}} \overline{\mathbf{y}}+\gamma_{\nu \mu} \overline{\mathbf{z}}, \quad \gamma_{\nu \mu} \equiv \sqrt{k^{2}-\left(\frac{\pi \nu}{L_{x}}\right)^{2}-\left(\frac{\pi \mu}{L_{y}}\right)^{2}}, \quad k=\frac{2 \pi}{\lambda} .
$$

We assume that the probe response is negligible outside the interval $|x| \leq L_{x},|y| \leq L_{y}$ for $z$ values of interest. [That is, $w(\mathbf{r})$ is a periodic extension.] To improve conditioning (see below), we include only propagating plane waves $\left(\gamma_{\nu \mu}\right.$ real) in the summation in (1). Evanescent waves ( $\gamma_{\nu \mu}$ imaginary) are exponentially attenuated and are negligible in the far-field region. We must also ensure that evanescent waves are not important contributors to the measured probe response; this is usually accomplished by maintaining a probe-totest-antenna separation of several wavelengths.

In matrix form (1) becomes

$$
\mathbf{w}=\mathbf{Q} \xi
$$

where $\mathbf{w} \equiv\left\{w\left(\mathbf{r}_{n}\right)\right\}, \mathbf{r}_{n}$ is the location of the $n$th measurement point, $\boldsymbol{\xi} \equiv\left\{\xi_{\nu \mu}\right\}$, and $\mathrm{Q} \equiv\left\{Q_{n, \nu \mu}=\exp \left(i \mathbf{k}_{\nu \mu} \cdot \mathbf{r}_{n}\right)\right\}$. The objective of near-field to far-field transformation is to

\section{U.S. Government Work Not Protected by U.S. Copyright}


determine the coupling product $\boldsymbol{\xi}$ from measurements w made in a restricted region near the test antenna.

In practical situations, where the number of measurements often exceeds the number of unknowns, the system (2) is overdetermined and will generally not have a solution. We will actually solve the normal equations

$$
\mathbf{A} \boldsymbol{\xi}=\mathbf{b}
$$

where

$$
\mathbf{A}=\mathbf{Q}^{H} \mathbf{Q}, \quad \mathbf{b} \equiv \mathbf{Q}^{H} \mathbf{w}
$$

The operator $\mathbf{Q}^{H} \equiv\left\{Q_{\nu \mu, n}^{H}=\exp \left(-i \mathbf{k}_{\nu \mu}^{*} \cdot \mathbf{r}_{n}\right)\right\}$ is the Hermitian (conjugate) transpose of Q. The solution $\boldsymbol{\xi}$ of (3) minimizes $\|\mathbf{w}-\mathbf{Q} \boldsymbol{\xi}\|$ (where $\|\mathbf{y}\|^{2} \equiv \mathbf{y}^{H} \mathbf{y}$ ); that is, this $\boldsymbol{\xi}$ is the least-squares estimate. Most methods for processing planar near-field data [based on the model (1)] solve (3), either directly or indirectly. In the standard plane-rectangular grid algorithm, $\mathbf{A}$ is diagonal and $\mathbf{Q}^{H}$ and $\mathbf{Q}$ can be applied with fast Fourier transforms, giving a computational complexity of $\mathcal{O}(N \log N)$. On the other hand, a direct solution using Gaussian elimination requires $\mathcal{O}\left(\hat{N}^{3}\right)$ operations. For typical problem sizes $\left(10^{4}<N<\right.$ $10^{6}$ ), the importance of computational efficiency is readily apparent.

\section{Conjugate Gradient Solution}

Because $\mathbf{A}$ is Hermitian and positive definite (assuming that $\mathbf{Q}$ is full rank) the conjugate gradient method is applicable. The algorithm is an iterative scheme which produces successive estimates $\boldsymbol{\xi}^{(j)}$. Initial estimates are not critical and we use $\boldsymbol{\xi}^{(0)}=0$ for simplicity. The relative error. (at the $j$ th iteration) is bounded by the residual $\mathbf{r}^{(j)}=\mathbf{b}-\mathbf{A} \boldsymbol{\xi}^{(j)}$ :

$$
\frac{\left\|\boldsymbol{\xi}^{(j)}-\boldsymbol{\xi}\right\|}{\|\boldsymbol{\xi}\|} \leq c^{2} \frac{\left\|\mathbf{r}^{(j)}\right\|}{\|\mathbf{b}\|} .
$$

Rate of convergence can be estimated with

$$
\left\|\boldsymbol{\xi}^{(j)}-\boldsymbol{\xi}\right\|_{A} \leq 2\left(\frac{c-1}{c+1}\right)^{j}\left\|\boldsymbol{\xi}^{(0)}-\boldsymbol{\xi}\right\|_{A} .
$$

Here $\|\mathbf{y}\|_{A}^{2} \equiv \mathbf{y}^{H} \mathbf{A y}$ and the condition number $c^{2}$ is the ratio of the largest to smallest eigenvalue of $\mathbf{A}$. (The condition number of $\mathbf{Q}$ is $c, c \geq 1$.) Thus, the conjugate gradient algorithm will always converge.

When condition numbers are large (poor conditioning), equations (4) and (5) indicate potential problems with computational accuracy and/ or convergence rate. Fortunately, it is often possible to improve conditioning by adding physically reasonable restrictions. For example, arbitrarily large condition numbers can arise when evanescent plane waves are included in the model (1). In the example of section 5 , the exclusion of evanescent fields results in an acceptable condition.

\section{Efficiency}

In the conjugate gradient procedure it is necessary to apply the matrix $\mathbf{A}=\mathbf{Q}^{H} \mathbf{Q}$ to a vector once each iteration. This can be done by a straightforward summation, but only in $\mathcal{O}\left(N^{2}\right)$ operations. In order to reduce complexity to $\mathcal{O}(N \log N)$ operations per iteration, we have developed a scheme that combines the unequally spaced fast Fourier transform with interpolation in $z$. For example, to apply $Q$ to $\xi^{(j)}$, we use the unequally spaced fast Fourier transform to evaluate (2) [in $\mathcal{O}(N \log N)$ operations] at the points $x_{n} \hat{\mathbf{x}}+y_{n} \hat{\mathbf{z}}+z \hat{\mathbf{z}}$ for several fixed values of $z$. We then use local interpolation in $z$ to reach the actual measurement locations $\mathbf{r}_{n}$. Since we are dealing with bandlimited functions, the numerical precision of the algorithm can be controlled and is specified as an input parameter. Computational time 
depends on the desired numerical accuracy and on the spatial distribution of data points. Our technique is most efficient when measurement locations lie close to a plane. Details will be presented elsewhere.

\section{Simulation}

We began with planar near-field data for a radiometer antenna with an aperture diameter of $25 \mathrm{~cm}$ and an operating frequency of $31.65 \mathrm{GHz}$. These data consist of 161 points in $x$ by 161 points in $y$ spaced by $\Delta x=\Delta y=0.38 \mathrm{~cm}(0.4 \lambda)$. A phase gradient was introduced into the near-field data to steer the main bearn $30^{\circ}$ from boresight. The model (1) was specified with $L_{x}=L_{y}=161 \times 0.38 / 2=30.59 \mathrm{~cm}$ and the coupling product was calculated using standard near-field to far-field transformation software. Position errors were then simulated by using (1) to calculate the probe response at nonideal measurement locations. In this setup, there are about 26000 simulated measurements and about 20000 unknowns (evanescent modes excluded).

Figure 1 shows the result of probe position correction when the position error at the grid point $n \Delta x \hat{\mathbf{x}}+m \Delta y \hat{\mathbf{y}}$ is given by

$$
\left(\begin{array}{c}
\Delta x \\
\Delta y \\
\Delta z
\end{array}\right)=\left(\begin{array}{c}
0.3 \cos (0.35 n) \cos (0.65 m) \\
0.3 \cos (0.25 n) \cos (0.15 m) \\
1.0 \cos (0.15 n) \cos (0.11 m)
\end{array}\right) \lambda .
$$

The peak magnitude of this position error is $1.1 \lambda$ and the rms magnitude is $0.52 \lambda$. The pattern computed ignoring probe position errors bears little resemblance to the correct pattern - the main beam is no longer recognizable. If we correct only for $z$ position errors, much of the true pattern is recovered. However, the gain is still about $1 \mathrm{~dB}$ low, and there are anomalous sidelobes. There is no discernible difference between actual and the fully position corrected patterns. Thus, three-dimensional position error correction can be important for steered-beam antennas.

For this example, the condition number is $c^{2} \approx 21,\left\|\mathbf{r}^{(9)}\right\| /\|\mathbf{b}\|<10^{-4}$, and $\left\|\mathrm{r}^{(29)}\right\| /\|\mathbf{b}\|<$ $10^{-8}$. Calculations were done on a $200 \mathrm{MHz}$ personal computer and required approximately 75 seconds per iteration.

\section{Summary}

A number of papers that treat nonideal measurement locations have been published (see, for example, [5]-[7]). We think that our approach compares favorably in terms of efficiency, accuracy, and simplicity. Major features are:

-The algorithm is iterative, with a fixed cost per iteration that is $\mathcal{O}(N \log N)$. The memory requirement is $\mathcal{O}(N)$ and is independent of the number of iterations.

- Convergence is guaranteed. Bounds [see (5)] on the convergence rate for the conjugate gradient procedure are tighter than for many alternative iterative techniques.

- Computational error (not measurement error) is bounded by the residual [see (4)].

- Our current implementation is fully three-dimensional.

- The recipe given in this paper is also applicable to cylindrical and spherical scanning geometries. The basic ingredient is an efficient procedure for predicting probe response at the measurement locations, based on an estimated modal spectrum.

The software is available from the authors. 


\section{References}

[1]R. C. Wittmann, B. K. Alpert, and M. H. Francis, "Planar near-field antenna measurements using nonideal measurement locations," Proc. Antenna Measurement Tech. Assoc., pp. $74-77,1996$.

[2]A. Dutt, and V. Rokhlin, "Fast Fourier transforms for nonequispaced data," SIAM $J$. Scientific Computing, vol. 14, pp. 1369-1393, 1993.

[3]G. Beylkin, "On the fast Fourier transform of functions with singularities," Applied and Computational Harmonic Analysis, vol. 2, pp. 363-381, 1995.

[4]D. M. Kerns, "Plane-wave scattering-matrix theory of antennas and antenna-antenna interaction," Nat. Bur. Stand. (U. S.) Monograph 162, 1981.

[5]L. E. Corey and E. B. Joy, "On computation of electromagnetic fields on planar surfaces from fields on nearby surfaces," IEEE Trans. Antennas Propagat., vol. AP-29, pp. 402$404,1981$.

[6]P. K. Agrawal, "A method to compensate for probe positioning errors in an antenna near-field facility," Proc. IEEE Antennas Propagat. Int. Symp., pp. 218-221, 1982.

[7]L. A. Muth, "General order $N$ analytic correction of probe-position errors in planar nearfield measurements," Proc. Antenna Measurement Tech. Assoc., pp. 331-335, 1995.

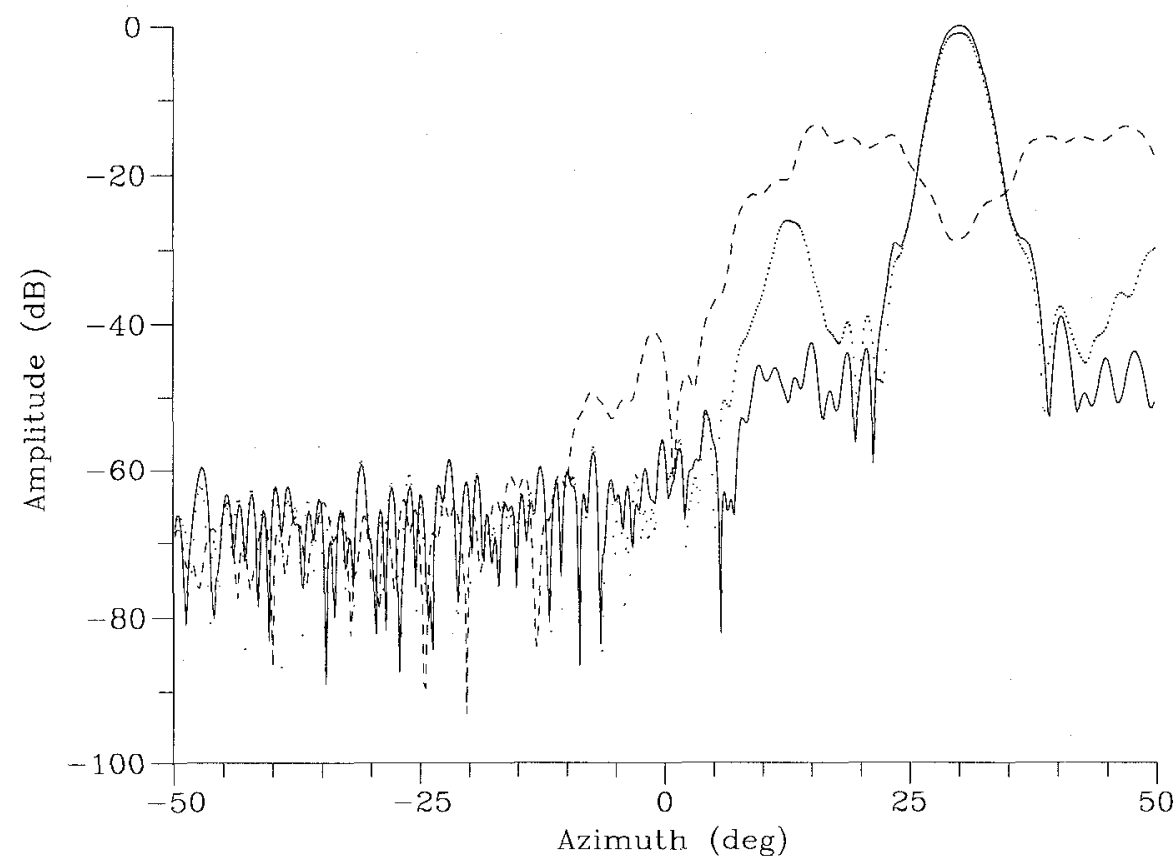

Figure 1: H-plane far-field pattern of the antenna with a steered beam. Probe position errors are given by eq (6). The solid line corresponds to the corrected and to the actual pattern. The dashed line shows the result of ignoring the position errors. The dotted line is the result of correcting for only the $z$ position errors. 